\title{
Roland Barthes' Bausteine zu einer Theorie des Interviews: Eine medienanalytische Reflexion
}

\author{
Natalie Binczek \\ Ruhr-Universität Bochum, Germanistisches Institut, \\ Universitätsstr. 150, 4478o Bochum \\ natalie.binczek@rub.de
}

\section{Das Interview}

„Ein Ursprung der Gesprächsform Interview lässt sich nicht eindeutig ausmachen", so Jens Ruchatz in der bislang einzigen umfassenden medienhistorischen Abhandlung zum Thema. ${ }^{1}$ Die Genealogie dieser spezifischen "Gesprächsform" verweist vielmehr auf unterschiedliche Felder und Disziplinen wie den Journalismus, die Soziologie oder Psychiatrie, die ihrerseits eigene Traditionen ausgeprägt haben und auf verschiedene Vorformen hindeuten, die letztlich nicht einen, sondern zahlreiche Ursprungsszenarien der „Gesprächsform Interview“ nahelegen. ${ }^{2}$ Auf das engste verknüpft mit dem Problem der historischen Rekonstruktion ist die Frage nach der Beziehung des Interviews zum Gespräch. Während Ruchatz dieses als übergeordnete Kategorie behandelt, folglich das Interview als eine bestimmte Form des Gesprächs auffasst und auf diese Weise in einen weitgespannten kulturhistorischen Horizont rückt, ${ }^{3}$ wird andernorts von einer dezidierten Differenz ausgegangen: Im Historischen Wörterbuch der Rhetorik etwa heißt es: „Vom Gespräch unterscheidet sich das Interview dadurch, dass die Sprechenden eine stark vorstrukturierte Rolle [...] übernehmen, statt sich wie im Gespräch initiativ und reaktiv abzulösen.4 ${ }^{4}$

Für die Geschichte des Interviews gilt, dass es sich spätestens seit dem 19. Jahrhundert einerseits als wichtiges methodisches Verfahren empirischer

1 Jens Ruchatz (2014), S. 16.

2 Ebd.

3 Siehe dazu auch: „Ein ,Interview ist ein Gespräch zwischen zwei oder mehr Teilnehmern, das sich durch eine ungleiche Rollenverteilung auszeichnet und in erster Linie zur Informationsbeschaffung dient." Seiler (2009), S. 403 .

4 Pekar (1998), S. 538. 
Datenerhebung in verschiedenen wissenschaftlichen Bereichen etabliert und andererseits als besondere journalistische Interviewtechnik ausdifferenziert hat. ${ }^{5}$ Die gebräuchlichen Definitionen als „Befragung im weitesten Sinn“ und als "publizistische Textsorte in Dialogform" deuten dabei auf die genannten Entwicklungsstränge hin, ${ }^{6}$ die ihrerseits weitere, gleichsam interne Differenzierungen hervorgebracht haben. So muss seine Bestimmung als ,publizistische Textsorte nicht nur die unterschiedlichen publizistischen Medien und die damit einhergehenden formalen Spezifikationen - neben der Zeitung und Zeitschrift auch den Rundfunk sowie die unterschiedlichen Online-Formate - berücksichtigen, sondern auch dem Umstand Rechnung tragen, dass die Presse bereits in der Mitte des 19. Jahrhunderts Interviews sowohl mit dem Anspruch, eine kritische Öffentlichkeit zu installieren, ${ }^{7}$ veröffentlichte, als auch zum Zweck der Unterhaltung, ${ }^{8}$ dann zumeist aus dem Bereich der ,Human Interest Stories. Allein auf den Journalismus bezogen lässt sich demnach aufgrund der verschiedenen medialen Formen und Praktiken des Befragens eine Vielfalt dessen ausmachen, was ein Interview sein kann. Zuweilen kommen auch Varianten journalistischer Befragung zum Vorschein, die Berührungspunkte zum wissenschaftlichen Interview erkennen lassen und die Ausgangstypologie letztlich vollends in Frage stellen.

\section{Das intellektuelle Gespräch}

Ungeachtet dessen, ob sie als eine spezifische Erscheinungsform des Gesprächs oder als eine von ihm zu unterscheidende Kategorie aufgefasst werden, implizieren und prozessieren Interviews eine asymmetrische Kommunikationsstruktur. Deshalb werden sie nicht nur in einer engen Beziehung zum Verhör gesehen, ${ }^{9}$ sondern, damit einhergehend, auch zur Macht. ${ }^{10}$ Denn

$5 \quad$ Siehe zum Interview in den unterschiedlichen Wissenskulturen: te Heesen (2013), S. 317328. Zur historischen Entwicklungen des journalistischen Interviews in der französischen Presse siehe: Kött (2004).

$6 \quad$ Pekar (1998), S. 533; Bentele (2007), S. 179.

7 Seiler (2009), S. 403.

8 „Die ersten journalistischen Interviews erschienen 1835 und 1836 im ,New York Herald; eingeführt wurden sie von James Gordon Bennett, dem Herausgeber des Blattes. In Europa setzten sich das Verfahren und die journalistische Textform nur langsam durch, erst ab Anfang des 20. Jhs. wurden Interviews zur Befragung bekannter Persönlichkeiten, insbesondere Politiker, üblich." Ebd., S. 180.

$9 \quad$ Kött (2004), S. $72 \mathrm{ff}$.

10 Die Befragung unter dem Gesichtspunkt der Macht untersucht Michael Niehaus (2003). 
die Praxis des Interviewens schreibe, der ihr verwandten Form des Verhörs vergleichbar, ihre Akteure in besondere Machtgefüge ein, die in der Regel den Fragenden dazu ermächtigten, den Befragten zur Rede zu stellen. Dass das Interview überhaupt als Instrument der bürgerlichen Öffentlichkeit und Kritik verhandelt wird, man denke hier nur an Brechts Überlegungen zum Rundfunk als Kommunikationsapparat(1932/33), ${ }^{11}$ hängt exakt mit dieser Machtimplikation zusammen: Das Interview stellt Machtstrukturen als Kommunikationsstrukturen aus und vice versa. Damit aber eröffnet es auch Möglichkeiten ihrer Umverteilung. Indes bildet die Autorität des zu Befragenden eine wichtige Voraussetzung des journalistischen Interviews. Ein Rederecht bekommt in der Regel nur eingeräumt, wer eine Stimme hat, die gehört werden soll und will; sei es nur, um sie schließlich zu demontieren. Diese Autorität bildet demnach in der Regel so etwas wie eine Zugangsberechtigung zumindest zum journalistischen Interview. ${ }^{12}$

Im intellektuellen Milieu der 1968er Jahre hatte Roland Barthes eine solche Stimme, die gehört werden wollte. Zahlreiche Interviews hat er gegeben. ${ }^{13}$ Ein beachtlicher Teil seines CEuvres liegt in Form von Gesprächen und Interviews vor, welche für unterschiedliche Medien, vor allem aber für den Rundfunk und für Zeitschriften, durchgeführt worden sind. Obzwar er immer wieder seine eigene Schreib- und Sprechpraxis in den unterschiedlichen Funktionskontexten seiner Arbeit - als Essayist, ${ }^{14}$ als Lehrer, als Professor und Vorlesender etc. - eingehend reflektiert und zum Teil theoretisch systematisiert hat, ${ }^{15}$ lässt sich eine Theorie des Interviews lediglich aus dem Umfeld seiner Interviewpraxis

11 Der Rundfunk als Kommunikationsapparat. Rede über die Funktion des Rundfunks. Für Brecht wird das Interview zum Instrument, mit dessen Hilfe die Funktion des Hörfunks von einer nur distributiven in eine kommunikative umgestaltet werden könnte: „Ich kenne sowenig wie Sie die Verpflichtungen etwa des Reichskanzlers, es ist Sache des Rundfunks, sie mir klarzumachen, aber zu diesen Verpflichtungen des obersten Beamten gehört es: regelmäßig durch den Rundfunk die Nation von seiner Tätigkeit und der Berechtigung seiner Tätigkeit zu unterrichten. Die Aufgabe des Rundfunks allerdings erschöpft sich nicht damit, diese Berichte weiterzugeben. Er hat über dies hinaus die Einforderung von Berichten zu organisieren, d.h. die Berichte der Regierenden in Antworten auf Fragen der Regierten zu verwandeln." Brecht (1992), S. 554.

12 Damit unterscheidet sich das journalistische und eine größere Publizität anstrebende Interview von solchen Ansätzen, wie sie in den Kulturwissenschaften etwa die Oral History vertritt. Ein Verfahren, welches selbst in literarische Projekte Einzug gehalten hat, was paradigmatisch in Runge (1968) umgesetzt wurde.

13 Siehe dazu auch den einführenden Kommentar zu Barthes' Interview mit Georges Charbonnier von Agathe Mareuge und Sandro Zanetti in diesem Heft.

14 Zum Hörfunk-Interview siehe Ruchatz (2014), S. $211 \mathrm{ff}$.

15 Zu Roland Barthes als Vorlesendem siehe Binczek (2016), S. 249-268. 
rekonstruieren. So verfasst er im Vorwort zur nachträglichen Veröffentlichung einer Reihe von zunächst im Rundfunk gesendeten Gesprächen ein Vorwort, ${ }^{16}$ das im Hinblick auf eine Theorie des Interviews programmatische Aussagen enthält. Dabei lässt Barthes das Vorwort mit einer dezidiert praxeologischen Beobachtung beginnen. Es ist gerade diese an den Verfahrensweisen orientierte Perspektive, die wichtige Anschlussstellen und Ausgangsbedingungen für den Entwurf seiner Interviewtheorie bietet:

Wir reden, man nimmt uns auf, eifrige Sekretärinnen hören unsere Äußerungen ab, säubern sie, schreiben sie nieder, interpunktieren sie, erstellen daraus ein erstes Skript, das uns vorgelegt wird, damit wir es aufs neue reinigen, bevor es der Veröffentlichung, dem Buch [...] überantwortet wird. ${ }^{17}$

Dass Barthes das Personalpronomen ,wir' im Eröffnungssatz dieses Vorwortes gebraucht, ist bereits ein erster Verweis auf die spezifische Schreib- und Sprechweise des Gesprächs bzw. Interviews, die er hiermit einleitet und reflexiv rahmt. Die Formulierung bezeichnet eine Kollektivität, die den Sprecher einbezieht, zumindest aber setzt das Personalpronomen zwei Gesprächspartner als Hauptakteure, ferner eine Aufzeichnungstechnologie, die das Gespräch reproduzierbar macht, sowie die es in ein „Skript“ übersetzenden Sekretärinnen voraus.

Unter dem Titel Dialogues, in welchem eben dieses mediale Ensemble zunächst ausgestrichen erscheint, wurden für den Radiosender France Culture Gespräche, die Roland Barthes mit Maurice Nadeau 1973 führte, aufgenommen, gesendet und im darauffolgenden Jahr in einer Nieder- bzw. Umschrift als Buch mit dem Titel Sur la littérature unter doppelter Autorschaft veröffentlicht. ${ }^{18}$ Der Titel der Sendereihe blendet also die Beteiligung anderer Akteure und Mittler aus. ${ }^{19}$ Suggeriert wird im Konzept der Dialogues eine kommunikative Symmetrie, wie sie auch das ideale Gespräch kennzeichnet: Zwei in Bezug auf ihre Funktionsrollen gleiche Personen tauschen sich in Wechselrede aus, um in einer den Widerspruch durchaus einbeziehenden Konversation ein gemeinsames Ergebnis zu erarbeiten. Es obliegt nicht dem einen, die Fragen zu

16 Zum Vorwort als Paratext siehe Genette (1992), S. $157 \mathrm{ff}$.

17 Barthes (2002), S. 9.

18 Barthes/Nadeau (1980).

19 „Mittler [...]“, so Bruno Latour, „übersetzen, entstellen, modifizieren und transformieren die Bedeutung oder die Elemente, die sie transportieren sollen. [...] Aus ihrem Input lässt sich ihr Output nie richtig vorhersagen." Latour (2010), S. 70. 
stellen, und dem anderen, sie zu beantworten. Die Struktur dieses Gesprächs folgt mithin nicht dem Schema des Interviews. Roland Barthes und Maurice Nadeau werden vielmehr mit der gleichen Frage nach dem Weg, dem Stand und der Entwicklung der Literatur konfrontiert: „Où va la littérature?" Eine einzige Frage, die seitens der Redaktion des Radiosenders an die beiden Gesprächspartner adressiert wird und die ausreicht, um ein längeres Gespräch, das keiner weiteren Vermittlung oder Moderation durch einen Interviewer mehr bedarf, anzustoßen. Eine Frage zudem, die das von Jules Huret 1891 ins Leben gerufene Projekt der Enquête sur l'évolution littéraire zitiert. ${ }^{20}$ Das Interviewsetting ist lediglich Initialzündung eines Gesprächs, das seine eigene Struktur entwickelt und von Anbeginn den Anschein erweckt, als habe es nichts mit einer journalistischen Öffentlichkeit zu tun, als sei es eine ausschließlich philosophische Begegnung. So geht Maurice Nadeau bereits in seinem ersten Redebeitrag darauf ein, dass die diesem Gespräch vorangestellte Frage ihrerseits literarisch-philosophischen Ursprungs ist: „Maurice Nadeau: Blanchot a écrit: ,Où va la littérature? Elle va vers elle-même.“" ${ }^{21}$ Nicht einmal mehr dem Anstoß der Unterhaltung wird die Urheberschaft einer Redaktion zuerkannt, sondern als Zitat Blanchots mit einer geistesgeschichtlichen Dignität versehen.

Das Gespräch beginnt, indem die Befragten die Ausgangsfrage auf ihren auktorialen Ursprung zurückführen, sie in Bezug darauf untersuchen, ob sie überhaupt sinnvoll formuliert ist und welche Implikation sie enthält. Weniger die Aufgabe, sie zu beantworten, als vielmehr diejenige, sie zu hinterfragen, steht im Fokus ihrer Aufmerksamkeit.

Roland Barthes: En toute honnêté, je ne comprends pas la formulation „Où va la littérature?", c'est déjà une question un peu spécieuse, comme toutes les questions prospectives. Et l'alternative voudrait dire quoi? La littérature et autres choses?22

Im Wechsel widmen sich die beiden Gesprächspartner seitenlang - Barthes beginnt und Nadeau folgt - der Reflexion der Frage, die ihren Austausch initiiert hat, bevor sie sich im Verlauf des Gesprächs hin und wieder auch gegenseitig Fragen stellen und ihrem eigenen Dialog so ein Strukturmoment des Interviews implementieren. Wenn Torsten Hoffmann und Gerhard Kaiser festhalten: „Eine kategoriale Trennung zwischen Interview und Gespräch würde

\footnotetext{
$20 \quad$ Siehe dazu te Heesen (2014), S. $138 \mathrm{ff}$.

21 Barthes/Nadeau (1980), S. 7.

22 Ebd.
} 
den Praxistest nicht bestehen “, ${ }^{23}$ dann bestätigt die hier beschriebene Konstellation ihre Aussage nachdrücklich. Zwar werden Barthes und Nadeau als Gesprächspartner eines ,Dialogs' in Szene gesetzt. Der Rahmen jedoch, innerhalb dessen dieser Dialog situiert und überhaupt erst ermöglicht wird, ist ein Interview. Ein Interview, das sich nach der ersten Frage schon in eine andere Form der Kommunikation wandelt. Denn dass die beiden Gesprächspartner überhaupt mit dieser Frage konfrontiert werden, geht auf ein journalistisches Setting des Befragens zurück. Die Frage nach dem ,Fortgang' der Literatur ist keine, die von den Kommunikanten selbst gestellt wurde, sondern sie ist von außen an sie adressiert worden. Indem die Befragten die ihnen gestellte Frage jedoch zum Gegenstand ihres Gesprächs machen, befragen sie den Fragesteller und kehren so das Rollenverhältnis um. Zudem stellen sie sich allmählich auch gegenseitig Fragen, womit sie die Rolle des Interviewers in gewisser Weise adaptieren und das Gespräch in ein Interview, sofern es als Befragung verstanden wird, übergehen lassen.

\section{Das Vorwort}

Diesem Interview-Gespräch zwischen Barthes und Nadeau ist ein Vorwort vorgeschaltet, welches allerdings nur einer der beiden verfasst hat. Bringt Barthes, der alleinige Verfasser des Vorwortes, deshalb das gesamte Gespräch unter seine Kontrolle? Verleibt er es seiner Autorschaft ein? Zumindest in der Buchfassung, deren Titel jede Dialogizität, Vermitteltheit und Bedingtheit zurücknimmt, um den Text stattdessen auf ein großformatiges Projekt hin zu transgredieren: In Sur la littérature wird durch die nur Barthes ausweisende Signatur des Vorwortes eine Schieflage zu dem ,wir' hergestellt, mit welchem es doch beginnt. Hier, im Vorwort, spricht und signiert eben nur einer, auch wenn er seinen Dialogpartner im Personalpronomen der Rede einbezieht. Muss in dem ,Wir' aber nicht auch die redaktionelle Stimme des Stichwortgebers und Fragestellers sowie die Mitarbeit der den Text aus der Mündlichkeit der Rede in die Schrift des Buchs übersetzenden Sekretärinnen mitgelesen werden? Obgleich in der Buchfassung des Gesprächs die Frage „Où va la littérature?“ nicht einmal mehr als eine redaktionell gestellte markiert ist, sondern nur noch als literarisches Zitat von den Gesprächspartnern Barthes und Nadeau verhandelt wird, erinnert das von Barthes verfasste Vorwort durchaus an die journalistische Entstehungsgeschichte und die den Text ermöglichenden Akteure.

23 Hoffmann/Gerhard (2014), S. 15. 
Es deutet auf das Zusammenwirken unterschiedlicher Mitspieler und Mittler ebenso hin wie auf den Umstand, dass das Buch die Rede einfriert.

Wir balsamieren unsere Rede wie eine Mumie ein, um sie zu verewigen. Denn man muß doch wohl seine Stimme ein wenig überdauern; man muß sie doch durch die Verstellung der Schrift irgendwo einschreiben. ${ }^{24}$

Barthes' Interesse am Interview gilt dem Aspekt der medientechnischen Operationen, die aus einem Gespräch einen Text machen, in welchem dieses eine neue, schriftlich fixierte Form annimmt und damit aufhört, mündliche Rede zu sein. Mittels der Transkription überdauert diese zwar, ${ }^{25}$ wird aber zugleich auch „verstellt", so die hier gewählte Formulierung. Vorausgegangen ist ihr eine Beschreibung, in welcher der Eingriff der Sekretärinnen mit dem Bildfeld der Reinigung ${ }^{26}$ und Säuberung verknüpft wird. „Wir reden, man nimmt uns auf, eifrige Sekretärinnen hören unsere Äußerungen ab, säubern sie, schreiben sie nieder", heißt es zu Anfang des Vorwortes. Aber auch die Redesubjekte nehmen diese Reinigungsarbeit auf, wenn sie ihrerseits die von den Sekretärinnen erstellten Transkriptionen „aufs neue reinigen“, um sie den Anforderungen einer Buchveröffentlichung anzupassen. Was sich anhand dieser Metaphorik als eine Art Entstörung zu erkennen gibt, die den Text von den ,Verunreinigungen“ der mündlichen Rede befreit, wird im weiteren Argumentationsgang als eine produktive, schaffende Tätigkeit bestimmt. „Beim Neuschreiben“, so Barthes weiter, womit er die schriftliche Übertragung als eine Operation begreift, welche einen neuen Text hervorbringt, „beim Neuschreiben dessen, was wir gesagt haben, schützen und überwachen wir uns, zensieren und streichen wir unsere Dummheiten, Selbstgefälligkeiten [...] oder unsere Unzulänglichkeiten [...].“27

Die ursprüngliche Rede wird auf der Grundlage ihrer technischen Aufzeichnung von anderen Beteiligten transkribiert und erst als Transkript den Sprechern zur Prüfung wieder vorgelegt. Parallel zu dieser als ,Reinigung' gekennzeichneten, gleichsam editorischen Bearbeitung, die sich zugleich auch als eine Form der Verschriftung konstituiert, vollzieht sich eine Produktion eigener Art, die Barthes als „Neuschreiben“ bestimmt, womit er einen Sprung von der zunächst nur reproduktiven Einrichtung des Textes zur produktiven Herstellung eines neuen anzeigt. Während die Bearbeitungen implizieren,

\footnotetext{
24 Barthes (2002), S. 9.

25 Siehe zu einer medienwissenschaftlichen Adaption und Erweiterung der Verfahren der Transkription Jäger/Stanitzek (2002).

26 Siehe dazu die Beiträge in Hahn/Ghanbari (2013).

27 Barthes (2002), S. $g$ f.
} 
dass lediglich unterschiedliche Varianten desselben Textes hervorgebracht werden, deutet das „Neuschreiben“ die Entstehung eines aus dem Bereich der unterschiedlichen (Transkript-) Fassungen scheidenden, eigenständigen Textes an - ein emergentes Ereignis. So hebt Barthes hervor, dass die nachfolgend gedruckten Ausführungen, die auf die Sprecherrollen von Roland Barthes und Maurice Nadeau verteilt werden und auf diese Weise eine unmittelbare Beziehung zu dem für den Hörfunk produzierten Gespräch suggerieren, vielmehr aus dem ursprünglichen Kontext herausgelöst sind.

Angesprochen ist damit ein Charakteristikum von Interviews überhaupt, dass sie nämlich, sofern sie aus dem Modus mündlicher Rede in den der schriftlichen Darstellung übertragen werden, auch einen neuen Text konstituieren. ${ }^{28}$ Dabei geht es nicht um bloße Übersetzungen der Rede in Schrift, sondern um die Herstellung von Kommunikaten, die den Bezug zum ursprünglichen Entstehungszusammenhang verlieren oder zumindest lockern. Was als Wiedergabe eines Gesprächs präsentiert wird, ist genau besehen das Resultat einer „Neuschreibung“. Es ist nicht die Reproduktion eines Gesprächs, was es übrigens auch nicht wäre, wenn kein Medienwechsel von mündlicher Rede in Schrift stattfände, denn auch bei der Postproduktion von Audiomaterial werden Eingriffe vorgenommen, welche die Kennzeichnung als „Neuschreibung“ legitimieren. Auch wenn Barthes ausschließlich die schriftlichen Bearbeitungen in den Blick nimmt, so stellt er dabei Effekte fest, welche sich ebenfalls auf andere mediale Mechanismen ausweiten lassen.

Der in der "Neuschreibung" hervorgebrachte Verweis auf die Ausgangssituation des Gesprächs legt eine Instabilität offen, die einen dreifachen Verlust hervorruft. Er betrifft erstens alle die Äußerungen, die mit den Eigenarten des jeweiligen Sprechens zusammenhängen und dabei mögliche Verzögerungen, Pausen, Umwege, semantische Sinnlosigkeiten etc. aufweisen. Eigenarten also, die nicht nur von den Standards schriftsprachlichen Ausdrucks abweichen, sondern zugleich auch den Sprechkörper als Medium der Kommunikation hörbar werden lassen. Ein auf Veröffentlichung angelegtes Interview, ein Dialog, der aufgezeichnet wird, um ihn im Rundfunk zu senden, hat von Anbeginn, bevor also die Mechanismen der „Neuschreibung“ greifen, etwas, so Barthes, ,Theatralisches'. Es wird als eine Inszenierung der öffentlichen Rede, im Fall der hier verhandelten Frage - „Où va la littérature?“ - sogar als eine Neuinszenierung der Enquête Jules Hurets und schließlich als eine Inszenierung des

28 „So schlägt sich im Geschriebenen ein neues Imaginäres nieder, nämlich das des ,Denkens'. Überall, wo die Rede mit dem Geschriebenen konkurriert, will Schreiben in gewisser Weise sagen: ich denke besser, nachdrücklicher; ich denke weniger für euch, ich denke mehr für die ,Wahrheit.“ Ebd., S. 12. 
Körpers bestimmt. Damit erteilt Barthes jeder Vorstellung einer Unmittelbarkeit des Sprechens eine Absage:

[N]icht, daß die Rede von sich aus frisch, natürlich, spontan, wahrhaftig und Ausdruck einer Art reiner Innerlichkeit wäre; ganz im Gegenteil, unsere Rede (vor allem in der Öffentlichkeit) ist unmittelbar theatralisch, sie entlehnt ihre Wendungen (im stilistischen wie im spielerischen Sinn des Wortes) einer ganzen Reihe von kulturellen und rednerischen Kodes; doch im Übergang zum Geschriebenen löschen wir gerade die Unschuld dieser Taktik aus. ${ }^{29}$

Hervorgehoben wird die rhetorische Faktur des Sprechens, die auf einer kulturellen Formung und Kodierung beruht. Ihr besonderer Effekt besteht gleichwohl darin, den Eindruck einer im Körper des Sprechenden verankerten Natürlichkeit zu evozieren. In der „Neuschreibung“ des Interviews bzw. Gesprächs werden überdies - womit Barthes den zweiten Verlust markiert alle sogenannten „Expletive des Denkens“, also bestimmte Füllwörter bzw. Partikel wie ,wohl', ,aber', , daher', die der Rede Nachdruck verleihen, jedoch eigentlich keinen „logischen Sinn“ haben, ${ }^{30}$ getilgt. Schließlich, so der dritte Verlust, wird das ,Phatische bzw. die ,Interpellationsfunktion' der unmittelbaren Adressierung des Gegenübers aufgehoben. Mit diesen Elementen der Sprache bezeichnet Barthes „Rufe, Modulationen - sollte ich, an Vögel denkend, sagen: Gesänge? - durch die ein Körper einen anderen sucht.“"

Mit den genannten ,Verlustkategorien' sind Aspekte angesprochen, welche auf die stilistisch-rhetorischen Differenzen zwischen dem ursprünglichen Gesprächstext und dem neu zu erstellenden abheben und dabei eine Fülle analytischer Möglichkeiten eröffnen, um die Textur eines Gesprächs zu spezifizieren. Dabei bleibt diese stets auf die Um- und Neuschreibungen verwiesen, welche für die anschließenden Veröffentlichungen an ihr vorgenommen werden. Sie bildet mit ihnen in gewisser Weise eine prozessuale Einheit. Hierin liegt das Entscheidende der Interview- bzw. Gesprächstheorie von Roland Barthes begründet. Indem er die Prozessualität der Bearbeitungen ins Zentrum rückt, definiert er das Interview bzw. Gespräch als eine Textform, welche die Einheit kollektiver Partizipation und die Einheit einer mehrfachen Umschrift, also einer operativen Kette, bildet. Umschriften bzw. „Neuschreibungen“, durch welche die Rede eine andere Gestalt annimmt, sich in etwas

$\begin{array}{ll}29 & \text { Ebd., S. 9. } \\ 30 & \text { Ebd., S. } 10 . \\ 31 & \text { Ebd., S. } 11 .\end{array}$


verwandelt, was Barthes als ein Hybrid und „spektakuläre[s] Gleiten von zwei Imaginären: dem des Körpers und dem des Denkens" definiert. ${ }^{32}$

Die Verschriftung führt nicht zu einer kompletten Löschung des Redebezugs bzw. - in der von Barthes vorgeschlagenen Metaphorik - ,des Körpers‘ aus dem Text. Dieser führt nicht nur das Denken vor. Vielmehr besteht die Spezifik eines schriftlich publizierten Gesprächs gerade in dem re-entry-Effekt der Mündlichkeit. Auf der Grundlage einer Textfassung, die von den schriftlich nur als Störung darstellbaren Momenten der Rede gereinigt ist und die sich mehr und mehr der Idee einer Repräsentation des Denkens anzunähern scheint, wird schließlich eine dem Körper - oder in anderer Terminologie: der Angesichtigkeit unter Anwesenden - korrespondierende Gesprächsstruktur hervorgebracht, welche der Schrift eigen ist. „Es geht darum“, so Barthes, „dem Leser eine Art Theater der intellektuellen Rollen, eine Inszenierung der Ideen darzubieten". ${ }^{33}$

\section{Der Text}

Unter einem Interview versteht man eine Form der Befragung von Personen mit ungleicher Rollenverteilung. ${ }^{34}$ Mit dieser Kennzeichnung wird die Beziehung zwischen den Interviewpartnern als maßgebliches Kriterium der Kommunikationsform Interview angesehen. Stets hat sie es diesem Verständnis zufolge mit dem Problem zu tun, wer wie zu befragen ist; immer wieder geht es darum, denjenigen, die nicht gehört wurden, eine Stimme zu geben, und diejenigen, die eine Stimme haben, zur Rede zu stellen, um ihre Stimme mitunter zu schwächen. Es geht um das kritische Potential, das der „ungleichen Rollenverteilung“ innewohnt und produktiv genutzt werden soll. Alle diese Aspekte, so scheint es, werden von Roland Barthes übergangen. Die Problematik der „ungleichen Rollenverteilung“ löst sich bei ihm in der unspezifischen Kollektivität des ,Wir', das dennoch ungleiche Beziehungen impliziert, förmlich auf. Es löst sich auch in der Replatzierung des Interviews durch ein philosophisches Gespräch zwischen zwei gleichwertigen Gesprächspartnern, Barthes und Nadeau, auf. In seinem Vorwort aber wendet Barthes sich den medientechnischen Bedingungen der Veröffentlichung sowohl des mündlichen Gesprächs als auch seiner nachträglichen schriftlichen Publikation zu.

\footnotetext{
32 Ebd., S. 12.

33 Ebd.

34 Seiler (2009), S. 403.
} 
Nicht die Personen, sondern die Schreibprozesse stehen im Zentrum seiner Aufmerksamkeit. Aus dieser Perspektive tritt die Einheit der Person, der befragten ebenso wie derjenigen, die die Fragen stellt, hinter die Beobachtung zurück, dass sich ihre Dynamik letztlich nur als ein textueller Prozess verstehen lässt.

\section{Literatur}

Barthes, Roland (2002): „Von der Rede zum Schreiben“, in ders.: Die Körnung der Stimme. Interviews 1962-1980. Frankfurt a. M.: Suhrkamp, S. 9-13.

Barthes, Roland/Nadeau, Maurice (1980): Sur la littérature. Grenoble: Presses universitaires.

Bentele, Günter (2007): „Interview“, in: Harald Fricke (Hg.): Reallexikon der deutschen Literaturwissenschaft, Bd. II: $H-O$. Berlin/New York: De Gruyter, S. 179-180.

Binczek, Natalie (2016): „Roland Barthes' Vorlesungsmanuskripte ,Die Vorbereitung des Romans' und das Engagement, eine Vorlesung zu halten“, in: Jürgen Brokoff/Ursula Geitner/Kerstin Stüssel (Hg.): Engagement. Konzepte von Gegenwart und Gegenwartsliteratur. Göttingen: V\&R unipress, S. 249-268.

Brecht, Bertolt (1992): „Der Rundfunk als Kommunikationsapparat. Rede über die Funktion des Rundfunks", in ders.: Große kommentierte Berliner und Frankfurter Ausgabe, Band 21 (Schriften 1). Hg. v. Werner Hecht. Berlin: Aufbau; Frankfurt a. M.: Suhrkamp, S. 552-557.

Genette, Gérard (1992): Paratexte. Das Buch vom Beiwerk des Buches. Frankfurt a. M./ New York: Campus.

Hahn, Marcus/Ghanbari, Nacim (Hg.) (2013): Reinigungsarbeit. In: Zeitschrift für Kulturwissenschaften 7,1 .

Hoffmann, Torsten/Kaiser, Gerhard (2014): „Echt inszeniert. Schriftstellerinterviews als Forschungsgegenstand", in dies. (Hg.): Echt inszeniert. Interviews in Literatur und Literaturbetrieb. Paderborn: Fink, S. 9-25.

Jäger, Ludwig/Stanitzek, Georg (Hg.) (2002): Transkribieren. Medien/Lektüre. München: Fink.

Kött, Martin (2004): Das Interview in der französischen Presse. Tübingen: Niemeyer.

Latour, Bruno (2010): Eine neue Soziologie für eine neue Gesellschaft. Einführung in die Akteur-Netzwerk-Theorie. Frankfurt a.M.: Suhrkamp.

Niehaus, Michael (2003): Das Verhör. Geschichte - Theorie - Fiktion. München: Fink.

Pekar, Thomas (1998): „Interview“, in: Gert Ueding (Hg.): Historisches Wörterbuch der Rhetorik, Bd. 4. Darmstadt: Wissenschaftliche Buchgesellschaft, S. 533-539.

Ruchatz, Jens (2014): Die Individualität der Celebrity. Eine Mediengeschichte des Interviews. Konstanz/München: UvK-Verl.-Ges. 
Runge, Erika (2008 [1968]): Bottroper Protokolle. Frankfurt a. M.: Suhrkamp.

Seiler, Sascha (2009): „Interview“, in: Dieter Lamping (Hg.): Handbuch der literarischen Gattungen. Stuttgart: Kröner, S. 403-407.

te Heesen, Anke (2013): „Naturgeschichte des Interviews“, in: Merkur 67, 4, S. 317-328. te Heesen, Anke (2014): „,Ganz Aug, ganz Ohr‘. Hermann Bahr und das Interview um 1800", in: Torsten Hoffmann/Gerhard Kaiser (Hg.): Echt inszeniert. Interviews in Literatur und Literaturbetrieb. Paderborn: Fink, S. 129-150. 